\title{
The implementation of the Wendelstein 7-X control an data acquisition concepts at VUVIXUV overview spectrometers HEXOS
}

\author{
Jörg Schacht ${ }^{*}$, Steffen Pingel ${ }^{*}$, Uwe Herbst ${ }^{*}$, Christine Hennig $^{*}$,Rainer Burhenn ${ }^{*}$, Klaus-Peter Hollfeld ${ }^{* *}$, Frank \\ Jordan $^{* *}$, \\ * Max Planck Institut für Plasmaphysik, Wendelsteinstrasse 1, 17491 Greifswald, Germany \\ *** Forschungszentrum Jülich GmbH, Wilhelm-Johnen-Straße, 52428 Jülich, Germany
}

HEXOS (high efficiency extreme ultraviolet overview spectrometer) is an optimized set of four efficient VUV/XUV spectrometers. It is suitable for a complete coverage of the wavelength range of interest with sufficient spectral resolution. The spectrometers cover the entire wavelength range of 2.5-160 $\mathrm{nm}$ with high performance (up to 9999 spectra at spectra rate of 1000/sec). To operate according to the Wendelstein 7-X (W7-X) control and data acquisition guidelines all necessary concepts for safety, autonomous and subordinated operation, and segment program controlled experiment operation will be implemented at HEXOS. The design of the HEXOS control and data acquisition system and the implementation of the main W7-X control and data acquisition concepts are described. An outlook on the test phase at the TEXTOR (Tokamak Experiment for Technology Oriented Research) device and the commissioning phase at W7- $\mathrm{X}$ is given.

Keywords: overview spectrometer, diagnostic, data acquisition, control system, segment control system,

\section{Introduction}

The most important parameters for a characterization of magnetically confined fusion plasma are the electron density, the electron temperature, the ion density and ion temperature, the plasma pressure, the total energy content, plasma currents, the radiated power, and the characterization of the plasma turbulences. Also the plasma properties are strongly linked to the content and transport of impurities which are either a result of plasma-wall contacts, or produced by fusion reaction, or even added on purpose via the gas inlet system to modify the plasma radiation characteristics or to diagnose plasma parameters. The measurement of the impurity species and its concentration in the plasma has two aspects, a safety issue (protection of the plasma facing components) and a diagnose issue (determine the impurity transport properties). Therefore, a set of four VUV/XUV overview spectrometers were developed for W7-X in cooperation between IPP, FZJ and Horiba Jobin-Yvon (France) [1]. The HEXOS spectrometers (high efficiency extreme ultraviolet overview spectrometer) cover the entire wavelength range of 2.5-160 $\mathrm{nm}$ with high performance.

Before final assembly at W7-X, the HEXOS's control and data acquisition system has to be setup fulfilling the established CoDaC (Control, data acquisition, and computing) concepts at W7-X.

After a short overview about the design of the spectrometer, the implementation of the main W7-X control and data acquisition concepts will be described.

The whole component HEXOS inclusive its segment control system and continuously data acquisition will be installed at TEXTOR for test operation prior to assembly at W7-X. Also, for this operation test a minimalistic W7-X CoDaC system infrastructure dedicated to (external) integration tests has to be installed at TEXTOR for a simulation of the W7-X central control system and its infrastructure.

\section{Spectrometer design}


The spectrometers of HEXOS cover a wavelength range of $2.5-160 \mathrm{~nm}$, divided into 4 subsections with some overlapping.

The optical path of spectrometers consist of the following elements: a throw-away filter with four filter positions, an entrance slit diffraction grating and a plane detector. The slit is horizontally and vertically adjustable via electrical drives (two step motor units). A continuous readout of the spectra at a high time resolution was required for the W7-X operation. Therefore an open Multi Channel Plate (MCP, Burle Industrie, Inc.) detector is used. The voltage between the MCP exit side and the phosphor screen is up to $8 \mathrm{kV}$. Four power supplies generate three different voltage stages for the MCPs. A MCP has a 40/25 mm fiber taper exit, which is directly coupled with the camera head. The camera is an NMOS linear image sensor C7883 by Hammamatsu with 1x 1024 pixels [2]. An electronic board generates all necessary control signals for the sensor chip. Also for a correct measurement of the charge of each sensor pixel the video output signal of the sensor is amplified and subsequently integrated by a fast switched integrator.

All four spectrometers are installed at one W7-X port to observe the same plasma volume. By twos the spectrometers share one beam line. The designated W7-X port has a gate valve, controlled by the W7-X central gate valve control system.

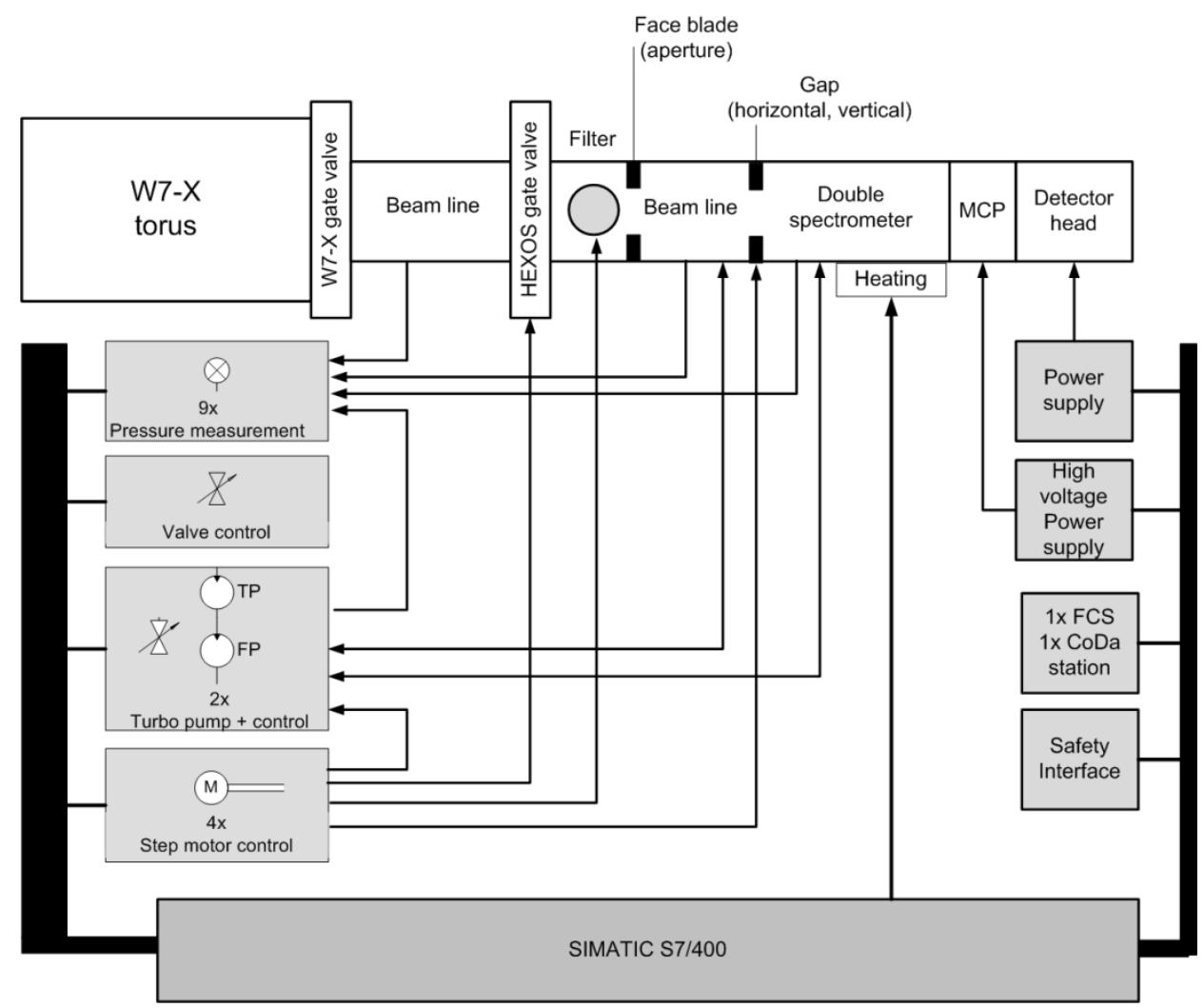

Fig. 1: Overview of HEXOS control and data acquisition

Two pumping stations, equipped with a roots and a turbo pump, are responsible for the vacuum condition into the grating and entrance slit chamber of the double spectrometers. A gate valve can isolate the W7- $\mathrm{X}$ torus volume from the volume of the spectrometer beam line. By a shutdown of the vacuum pumps the chamber of the spectrometers must be flooded with nitrogen gas. In total 9 pressure sensors are installed for observing the pressure in the beam line for three pressure section.

For control and data acquisition purposes a Fast Control Station (FCS), a Data Acquisition Station (DAQ Station) and a Programmable Logic Controller (PLC) are installed. An overview of the main data acquisition and control components for one double spectrometer is shown in Fig. 1.

\section{Layout of the HEXOS control system}


The layout of the control system for HEXOS has been designed to fulfill the W7-X rules for a diagnostic component of a middle complex type [3]. The HEXOS control system is responsible for controlling both data acquisition as well as control of the actuators necessary for the operation of the spectrometers. In principal the control system allows to operate HEXOS in two operational modes. The first mode is the stand-alone mode. This mode is mainly used for commissioning, tests and preparation of HEXOS for automatic control. Depending on the actual situation and the given working task in stand-alone mode the operator has the choice to control HEXOS manually by using the WinCC user interface. More complex sequences can be performed by means of pre-defined (HEXOS group) segment programs for the component HEXOS under control of the local segment control. For the second operation mode HEXOS must be sub-ordinate under the central control system. The central control system coordinates the control systems of the component HEXOS and also of all other sub-ordinate components during experiments. In this case the experiment runs are defined by sequences of segments, whereat a (W7-X project) segment contains the parameter sets for all components, which are involved during these experiment runs. A simple layer model of the control system of HEXOS is shown in Fig. 2.

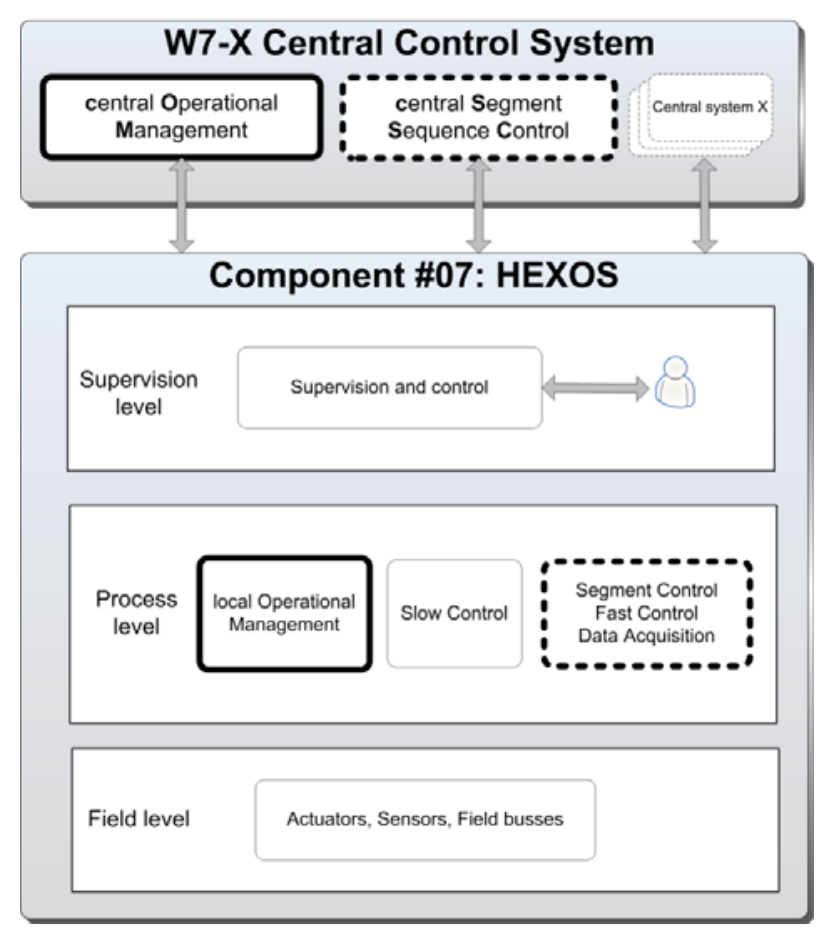

Fig.2: Layer model of the control system of HEXOS

The three layers: I. field level, II. process level, and III. supervision level are used for a structuring of the different tasks for data acquisition, control, data processing, and for the Human Machine Interface (HMI).

\section{1. Field Level}

The field level contains all controllable (e.g. high voltage power supplies, valves, and switches) and non-controllable elements (e.g. sensors for pressure measurements) of the two double spectrometers of HEXOS.

Every element of a given type has its own description, consisting of the following parts:

Element description (for the element High Voltage Power Supply):

- Object name : Element_PS_MCP,

- Element is member of group: spectrometer 1,

\section{Element attributes:}

- Operational state of element: e.g. ElOpState_ON,

- Authority to control the element, 
- $\quad$ Actual set values for voltages V1, V2, and V3,

- Limit values (stages: tolerance, warning, alarm) for the output voltages V1, V2, and V3,

\section{Element functions:}

- $\quad$ Swich ON main switch of MCP power supply,

- Swich OFF main switch of MCP power supply,

- Enable output of MCP-power supply,

- Disable output of MCP-power supply,

- $\quad$ Set the actual set values for the output voltage V1, V2, and V3,

\section{Enable / Disable attributes:}

- Enable status for dedicated element functions: e.g. Enable_PowerSupply_SwitchON,

- Disable status for dedicated element functions: e.g. Disable_Output_deactivate,

Definition of conditions for an integration of this element into its group (e.g. group spectrometer 1):

- Condition\#1: Operational state of element must be "ON",

- Condition\#2: no error, no warning,

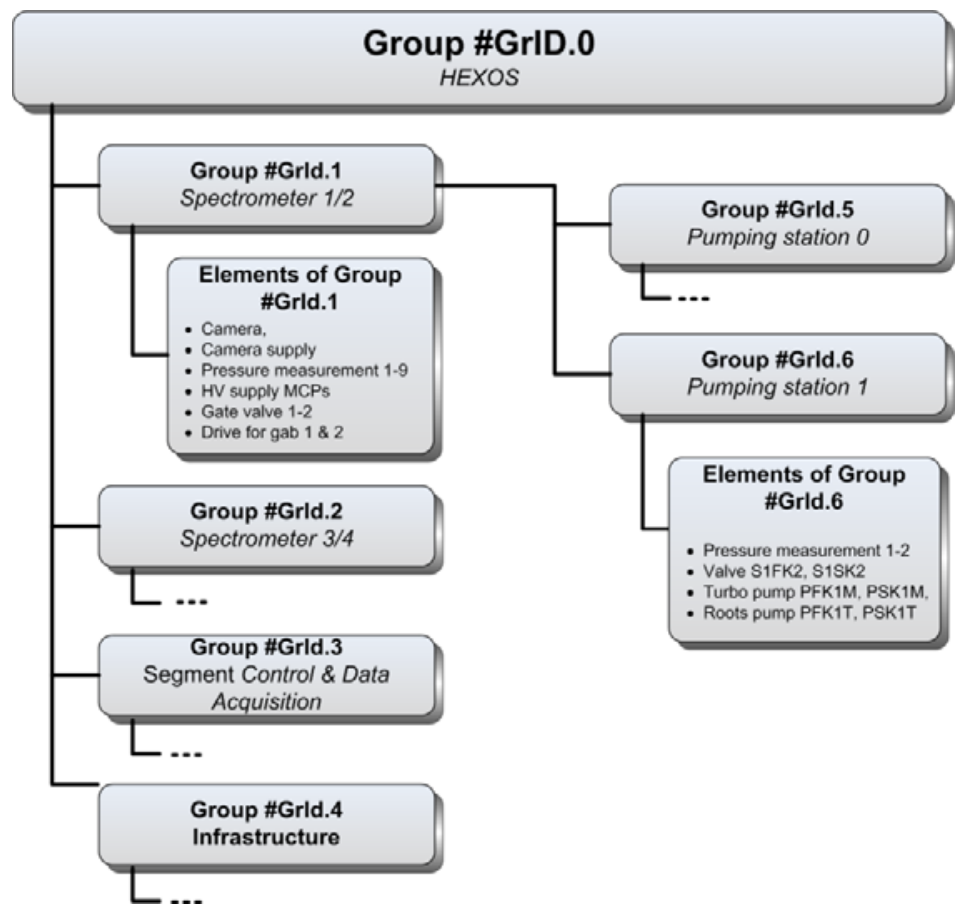

Fig. 3: Group structure for HEXOS

A hierarchical structuring of all elements of HEXOS has been done [see Fig. 3]. Every element of HEXOS is a part of a group, and a group itself can also contain other groups. Groups have a similar description like elements. For HEXOS there have been defined 7 groups, in which the group 0 represents the highest level of aggregation for groups and elements. The definition of elements and groups is an effort, but it offers a wide range of advantages. This kind of structuring allows making a clear arrangement of all elements of HEXOS corresponding to their functions and linking, the definition of interactions, a hierarchical error treatment, a simplification due to definition of complex operations, and is a basis for a good design of the HMI. Another important aspect is a re-use of already defined and tested groups and elements from other implemented installations.

\subsection{Process Level}

The process level of HEXOS summarizes the operational management (OPM), the slow control and the segment 
controlled data acquisition and fast control stations. Three types of controllers are used for different control and data acquisition purposes. For slow control processes and for the OPM a Siemens SIMATIC S7 PLC is used. The component operational management is responsible for the preparation of the component HEXOS for operation. Control processes with real time requirements are done using a Fast Control Station, i.e. a 19" PC running the VxWorks operating system. The real time software is being developed in C++. The data acquisition of the 4 spectrometer cameras is implemented on another 19" PC (DAQ station) with a PXI bus expansion PCIE 8560-PXI 8565 and a Analogue to Digital Converter (ADC) card (NI 6250, National Instruments) per detector. The ADC card NI PXIe-6124 S Series is a multifunction data acquisition module and offers simultaneous sampling at $1 \mathrm{MS} / \mathrm{s}$ per channel, 16 bit resolution. The sequence of data acquisition of the four CCD detectors is shown in Fig. 3. A pulse generator produces the clock signal $\left(f_{c \mid k}=4096 \mathrm{~Hz}\right)$ and a gate signal for controlling the read out process of the 1024 sensor pixels. The outputs of the detectors are amplified and subsequently integrated by fast switched integrators in order to calculate the charge that has been collected in a pixel. Four ADCs sample the analogue video output signals of the detector electronic. The maximum spectra rate is $1000 \mathrm{~s}^{-1}$ by 1024 pixel/spectra and 16 bit resolution for the measurement values.

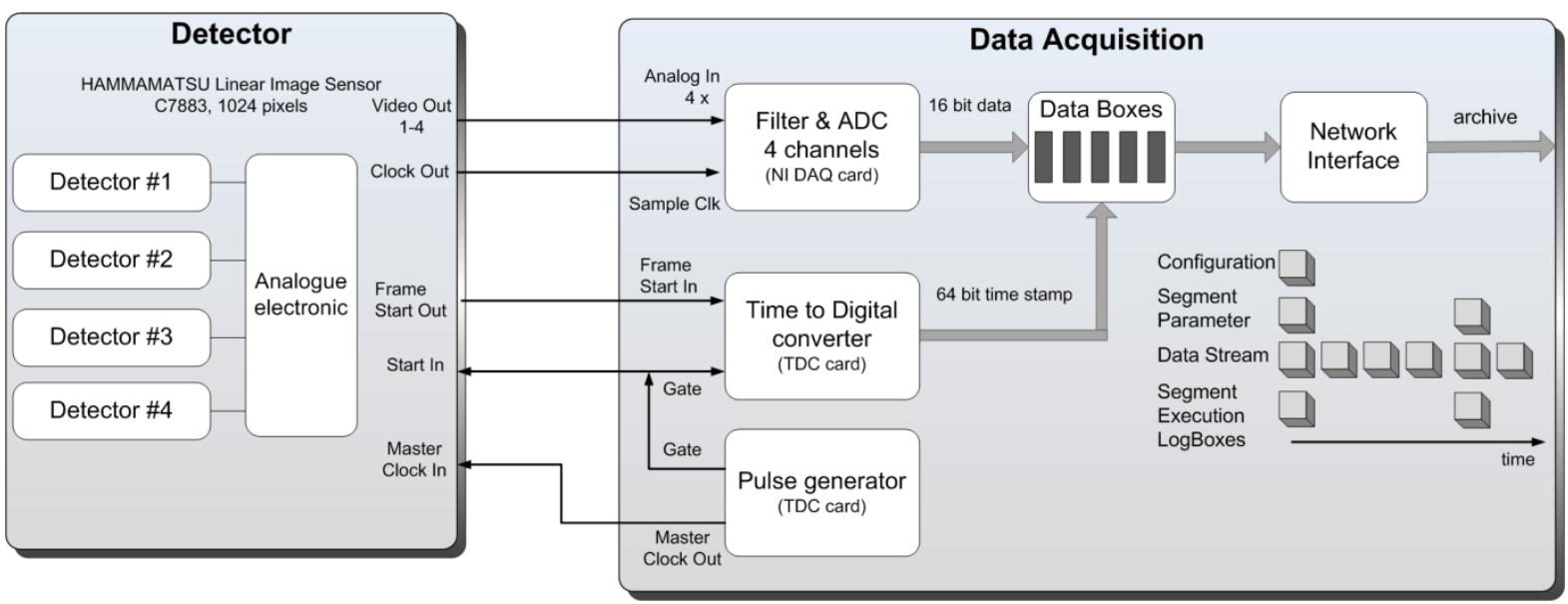

Fig.4: Data acquisition of the spectrometer cameras

A 64 bit time stamp for every frame is produced by the time capture hardware of the time to digital converter (TDC card). The data acquisition is running in a continuous operation mode. The produced data stream, the configuration of the FCS and DAQ station, the actual segment parameters and a protocol for the segment sequence execution (Segment Execution LogBoxes) during a experiment run have to be stored in the archive and configuration data base [7].The FCS and the DAQ station are equipped with special software modules responsible for generating the control objects for segment control purposes dependent on the actual experiment program, switching between these objects in real time and controlling the component by means of the active objects.

The behavior of the HEXOS during experiment runs is fundamentally determined and defined by the parameter sets of the segments. All segment controlled objects of the control stations needs parameter entries for all segments. In principle all these parameters can be changed from one segment to the next segment. But from the point of view of a diagnostician, the behavior of HEXOS during experiment runs can fully be described by a sub-set of these segment parameters - the high level segment parameter- and the remaining part is set by using default values defined within a so called pattern segment. The use of high level parameter allows a more physic oriented view on the diagnostic. Transformation functions are used to transform of the high level parameters into the pre-set definitions of the low level segment parameters. For the first operational period of HEXOS the following high level parameters have been identified to be settable in a segment program:

- Position of the filter wheel 1,2,3, and 4,

- Gap high for spectrometer 1, 2,3, and 4,

- $\quad$ Gap width for spectrometer 1, 2,3, and 4,

- MCP voltages V1, V2, and V3 for spectrometer 1, 2,3, and 4,

- Frame frequency for read-out the spectrometer camera 1, 2,3, and 4, 
- $\quad$ Archiving of spectrometer 1,2,3, and 4 data mandatory: Yes/No,

In the actual configuration the FCS has to realize the transfer of the set values given by the segment definition for the PLC controllable elements. A later version of HEXOS segment control system should extend the possibilities of the FCS with a feedback control loop to prevent overexposure of the spectrometer cameras and allow life supervision of specific plasma impurities.

Another important task of the FCS is the communication with the central segment sequence controller and with the PLC of the local OPM of HEXOS. An overview about the standard communication between the different controllers of HEXOS and with the central control system is given in [4].

Every control system has to be synchronized with the time of the central Trigger Time event system (cTTE system). The PLC uses the Simple Network Time Protocol for this purpose and reaches time accuracy in the range of $\geq 10 \mathrm{~ms}$. The requirements regarding the accuracy of time for time stamping of measurement values are significantly higher and therefore the control stations are equipped with special hardware time synchronization, triggering, time stamping and event handling $[5,6]$.

A risk analysis for HEXOS operations has justified the necessity of an own safety system. This system guarantees the safety of both personnel and environment for the component HEXOS. The safety system of the central control system handles interactions between components, the environment, and personnel. A standard interface module is responsible for exchange of safety signals between HEXOS and the central safety system. It provides an enable signal for HEXOS high voltage supply depending on the W7-X machine state like off, standby, or experiment. If the operational state "W7-X Emergency Stop" is active, the corresponding safety signal immediately switches the HEXOS operational state into the "Emergency OFF" state. The safety state of HEXOS is signalized by the safety signal "Safe State of HEXOS".

\subsection{Supervision Level}

The third level of the layer model describes the tasks for control and supervision of the HEXOS control system and the realization of the HMI interface. For visualization of these systems the Siemens WinCC program in a client-server configuration has been chosen. The hierarchical structure of the pages for the visualization is the same for all W7-X components. The visualization starts on the top level with the component overview, which contains important status information of the component (e.g. operational state of HEXOS, Alarms and warnings, status "Authority to control", status "Ready for segment operation") and of the whole W7-X device (e.g. operational state of W7-X). In the middle part of this overview page a schema of the two double spectrometers is placed. The user can get more detailed information about the elements and actual measurement values of one double spectrometer from the page "Spectrometer 1, 2" respectively from page "Spectrometer 3, 4". The look and feel and the operation of the elements are standardized for all WinCC applications of the W7-X diagnostic components.

\section{Outlook}

A test of the W7-X CoDaC component HEXOS at the tokamak TEXTOR is planned for October 2011. For these tests a test environment is necessary. For a simulation of all important central control components a so called "Mobile CoDaC" system is under development. The "Mobile CoDaC" contains all necessary IT infrastructure in a box (e.g. configuration, archive and segment program data bases), CoDaC infrastructure (e.g. networks, power supplies, and console), and the central control system (central segment sequence control system, central timing system, application software for experiment control and visualization). After the test phase at TEXTOR the whole HEXOS component will be transferred to IPP Greifswald for the final installation and commissioning.

\section{References}

[1] W. Biel, G. Bertschinger, R. Burhenn, R. König; Design of a high-efficency extreme ultraviolet overview spectromter system for plasma impurity on the stellarator experiment Wendelstein 7-X; Review of scientific instruments, Volume 75, Number 10, October 2004.

[2] NMOS linear image sensor C7883 by Hammamatsu, www.hammamatsu.com. 
[3] J. Schacht, H. Niedermeyer, H. Laqua, A. Spring, S. Pingel, A. Wölk, Tasks and structure of the WENDELSTEIN 7-X control system, Fusion engineering and design, Volume 81, Issues 15-17, July 2006.

[4] J. Schacht, H. Laqua, M. Lewerentz, A. Spring, S. Pingel, G. Kühner, Standardized Communication in the Control System of the Experiment WENDELSTEIN 7-X, IEEE Transactions on Nuclear Science, Volume 55, Feb. 2008.

[5] J. Schacht, H. Niedermeyer, H. Laqua, "The Trigger-Time-Event System for the W7-X Experiment", Proceedings of the 12th IEEE Real Time Conference on nuclear and plasma science, Valencia, June 2001, S.240-244.

[6] J. Schacht, H. Laqua, H. Niedermeyer, "Synchronization of processes in a distributed real time system exemplified by the control system of the fusion experiment WENDELSTEIN 7-X, Proceedings of the 14th IEEE Real Time Conference on nuclear and plasma science, Stockholm, May 2005.

[7] P. Heimann, S. Heinzel, Ch. Hennig, H. Kroiss, G. Kühner, H. Kühntopf, J. Maier, J. Reetz, M. Zilker, "The data acquistion system of W7X", Proceedings of the 3rd IAEA Technical Commitee Meeting on Steady-State Operation of Magnetic Fusion Devices, 2-3 May 2002, Greifswald, Germany, 6-7 May 2002, Arles, France. 